\title{
Aspectos clínicos e epidemiológicos da leishmaniose visceral na região do baixo Tocantins/PA
}

A Leishmaniose Visceral é um agravo com grande impacto para a saúde pública, e que no Brasil foi responsável por $96 \%$ das notificações que ocorreram nas Américas em 2017, o que justifica a proposta desse trabalho. Por tanto, o estudo objetivou descrever os aspectos clínicos e epidemiológicos da Leishmaniose Visceral na região do Baixo Tocantins, área endêmica no Estado do Pará. Como técnica de abordagem, a pesquisa apresenta um estudo transversal, descritivo, retrospectivo, sobre a base de dados do SINAN, no período de 2007 a 2015, referente aos casos de Leishmaniose Visceral em pacientes maiores de 18 anos, notificados nesta região. Foram identificados 284 casos da doença em pacientes com 18 anos ou mais, de um total de 1267 casos notificados no período. Houve predomínio da doença em indivíduos do sexo masculino, entre 18 e 27 anos, pardos, com ensino fundamental incompleto. 0 diagnóstico demorou de 0 a 90 dias, entre os primeiros sintomas e a notificação dos órgãos responsáveis e a maioria dos pacientes teve uma evolução para cura. 0 espaço de tempo, entre o diagnóstico e o início do tratamento, tem implicação direta no desfecho clínico do paciente. Dessa forma, estudos como este comprovam a necessidade de treinamento constante das equipes de saúde para a melhor identificação de casos de Leishmaniose Visceral.

\section{The clinical features and epidemiology of visceral leishmaniasis in the region of the lower Tocantins/PA}

\begin{abstract}
The Visceral Leishmaniasis is a condition with a major impact on the health of the public, and that in Brazil is responsible for $96 \%$ of the notifications that have occurred in the Americas by the year 2017, and the reason for the proposal of this work. Therefore, the study aimed to describe the clinical features and epidemiology of Visceral Leishmaniasis in the region of the Lower Tocantins river, an area that is endemic in the State of Pará, brazil. As a technique, an approach, and the research has a cross-sectional study is a descriptive, retrospective study based on the data in the SINAN, in the period from 2007 to 2015 , which refers to the cases of Visceral Leishmaniasis in patients older than 18 years old, have been reported in the area. We identified 284 cases of a disease in a patient 18 years of age or older, out of a total of 1267 cases in the same period. There was a predominance of the disease in male individuals, aged between 18 and 27 years of age, brown-skinned, with a primary education level. The diagnosis took a 0-to-90 days between onset of symptoms and notification of the bodies responsible, and the majority of the patients improved in order to heal. The length of time between diagnosis and initiation of treatment, has a direct implication in the clinical outcome of the patient. In This way, studies such as these demonstrate the need for ongoing training of health-care workers to identify cases of Visceral Leishmaniasis.
\end{abstract}

Keywords: Prevalence; Kala azar; The outcome; Studies of the time series; Endemic.

Topic: Epidemiologia e Saúde Ambiental

Reviewed anonymously in the process of blind peer.
Nina Pinto Monteiro (D)

Universidade Federal do Pará, Brasil http://lattes.cnpq.br/0187171562629768 http://orcid.org/0000-0002-4210-7039 ninapinto @hotmail.com

Rita Thaise Moraes Costa (iD Universidade Federal do Pará, Brasil http://lattes.cnpq.br/2148456702089608 http://orcid.org/0000-0002-5196-3478 rita.thaise@hotmail.com

Paula Caroline Coelho Fonseca (iD) Universidade Federal do Pará, Brasil http://lattes.cnpq.br/2580700084110521 http://orcid.org/0000-0002-0527-1024 paulacoelho.f@hotmail.com

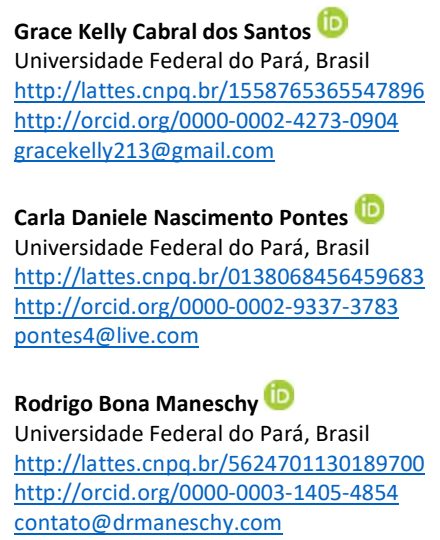

Clea Nazaré Carneiro Bichara (it) Universidade Federal do Pará, Brasil http://lattes.cnpq.br/2161704040280760 http://orcid.org/0000-0002-2995-0136 cleacarneirobichara@gmail.com
Referencing this:

MONTEIRO, N. P.; COSTA, R. T. M.; FONSECA, P. C. C.; SANTOS, G. K. C.; PONTES, C. D. N.; MANESCHY, R. B.; BICHARA, C. N. C.. Aspectos clínicos e epidemiológicos da leishmaniose visceral na região do baixo Tocantins/PA. Revista Ibero Americana de Ciências Ambientais, v.11, n.6, p.224-233, 2020. DOI: http://doi.org/10.6008/CBPC2179$\underline{6858.2020 .006 .0019}$ 


\section{INTRODUÇÃO}

A Leishmaniose Visceral é uma zoonose causada por protozoários do gênero Leishmania, a espécie mais prevalente no Brasil é a Leishmania chagasi, transmitida pela picada da fêmea do mosquito flebotomíneo Lutzomyia longipalpis que envolve os cães como reservatórios (MACHADO et al., 2016). O cão é o principal reservatório doméstico do parasita para os seres humanos e a principal fonte de infecção para os flebotomíneos nas áreas urbanas, mas já foram relatados casos de infecção em gatos, canídeos selvagens e cavalos (SANCHES et al., 2016).

A (LV) é a forma clínica mais grave dentre os tipos de leishmanioses, mediante suas possíveis complicações e potencial evolução para óbito (OPAS, 2017). A doença está presente nos cinco continentes, entretanto, mais de $90 \%$ dos casos ocorrem em apenas seis países: Índia, Bangladesh, Sudão, Sudão do Sul, Brasil e Etiópia. (ALVAR et al., 2012). Dados recentes mostraram que 96\% dos casos novos de LV que ocorreram nas Américas, foram registrados no Brasil (OPAS, 2017). Em 2013, o Estado do Pará foi o terceiro no Brasil, em número de ocorrência de LV, com 273 casos (BRASIL, 2014a) e 14 registros de óbitos (BRASIL, 2014b).

Estudos mostraram que essa enfermidade vem passando por diversas alterações ao longo do tempo, sob o impacto das constantes modificações urbanas, devido, principalmente, a mudança na interação entre homens, ambiente e processos biológicos, ocasionada pelo crescimento de megacidades, deslocando as pessoas para áreas com condições precárias de moradia, saneamento e infraestrutura. De modo geral, o movimento migratório da população rural para as periferias da cidade, ocasionou um inchaço populacional na malha urbana e, assim, o deslocamento do reservatório doméstico e a adaptação das espécies vetores a esses ambientes (MACHADO et al., 2016; REIS et al., 2017).

Essas alterações ajudam a identificar as constantes modificações que algumas doenças vêm sofrendo em seu processo evolutivo no tempo. Assim, pode-se perceber que epidemias urbanas de LV foram descritas em várias cidades do Brasil, como no Rio de Janeiro (RJ), Corumbá (MS), Belo Horizonte (MG), Santarém (PA), Araçatuba (SP), Palmas (TO), Três Lagoas (MS), Campo Grande (MS), dentre outros (BRASIL, 2014c). Dados de Reis et al. (2017), adquiridos através do estudo do SINAN, no período de 2001 a 2014, demostrou que 70\% dos casos no Brasil ocorreram em residentes de áreas urbanas.

Diante deste preocupante cenário é que se apresenta a questão norteadora da pesquisa intitulada aspectos clínicos e desfecho de evolução da Leishmaniose Visceral em adultos, região do Baixo TocantinsPará, uma vez que a LV no mundo e, pela relevância que este agravo endêmico tem para a Saúde Pública Nacional e Regional, mantendo seu potencial de letalidade e avançando com perspectiva de urbanização (SILVA JUNIOR et al., 2014).

Pelo supracitado, este estudo tem por objetivo descrever os aspectos clínicos e terapêuticos da Leishmaniose Visceral, na Região do Baixo Tocantins no Estado do Pará. Desse modo, será possível melhor compreender este fenômeno saúde-doença e meio ambiente, mas também, fundamentar as recomendações das medidas de prevenção, diagnóstico e intervenção terapêutica precoce, para que se obtenha um desfecho 
favorável na grande maioria desses pacientes.

\section{METODOLOGIA}

\section{Tipo de estudo}

Trata-se de um estudo transversal, descritivo e retrospectivo, em que foram analisados os dados secundários da Base de dados do SINAN, contidos nas fichas de notificação, referente à LV no período de 2007 a 2015 na região do Baixo Tocantins - PA.

\section{Local de estudo}

A área estudada correspondeu a Região do Baixo Tocantins que abrange uma extensão territorial de 36.024,20 Km² e inclui 11 municípios - Abaetetuba, Acará, Limoeiro do Ajuru, Moju, Tailândia, Barcarena, Baião, Cametá, Igarapé-Miri, Mocajuba e Oeiras do Pará (BRASIL, 2015) (Figura 1).

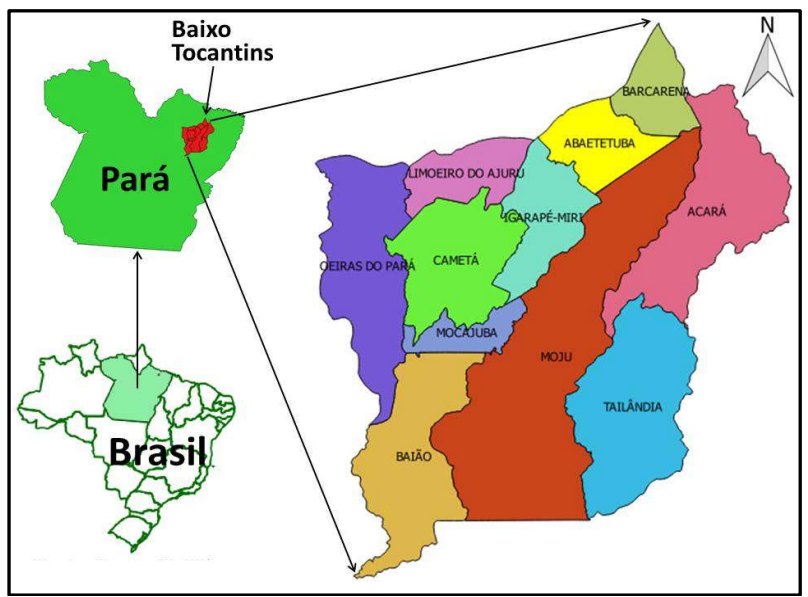

Figura 1: Mapa da Região do Baixo Tocantins no Estado do Pará.

\section{População e período de referência}

Os dados foram obtidos a partir da Base do Sistema de Informações de Agravos (SINAN) estadual, no período de 2007 a 2015, referente à LV na região descrita anteriormente. A amostra foi de pacientes maiores de 18 anos, representando 100\% dos casos de LV, que foram notificados no SINAN, no período descrito, pertencentes a microrregião do Baixo Tocantins. Foram excluídos todos os pacientes menores de 18 anos, e que não residiam na área citada.

\section{Análise de dados}

Os dados foram submetidos à análise estatística descritiva, para elaboração de gráficos e tabelas. 0 software empregado foi o Microsoft Excel 2016.

\section{Aspectos éticos}

Seguindo a resolução 466/12, do Conselho Nacional de Saúde, o estudo foi aprovado pelo Comitê de Ética Médica do Hospital de Clínicas Gaspar Viana, conforme parecer de número: 79912417.0.0000.0016. 


\section{RESULTADOS}

No período de análise foram notificados 1267 casos humanos de LV na Região do Baixo Tocantins, onde 284 (22,42\%) estavam acima de 18 anos, o que caracterizou a amostra do estudo. Percebeu-se um maior número de casos em 2008 e 2011, contudo, houve uma redução nos últimos 3 anos, podendo melhor ser exemplificado na Tabela 1.

Tabela 1: Distribuição de casos de Leishmaniose Visceral em adultos na região do Baixo Tocantins - PA, no período de 2007-2015.

\begin{tabular}{lll}
\hline Ano & $\mathbf{N}$ & $\%$ \\
\hline \hline 2007 & 37 & 13.03 \\
2008 & 47 & 16.55 \\
2009 & 31 & 10.92 \\
2010 & 32 & 11.27 \\
2011 & 41 & 14.44 \\
2012 & 32 & 11.27 \\
2013 & 24 & 8.45 \\
2014 & 21 & 7.39 \\
2015 & 19 & 6.69 \\
\hline \hline Total Geral & $\mathbf{2 8 4}$ \\
\hline \hline
\end{tabular}

Quanto ao perfil sócio-demográfico (Tabela 2), observa-se o predomínio do gênero masculino ( $n=225 ; 79,23 \%)$, com destaque a faixa etária entre 18 a 27 anos ( $n=100 ; 35,21 \%)$, sendo a maioria parda ( $n=204 ; 71,83 \%)$. Em relação a escolaridade, foi maior os pacientes com ensino fundamental incompleto $(n=$ $132 ; 60,15 \%)$.

Tabela 1: Perfil sócio-demográfico de pacientes adultos com Leishmaniose Visceral na região do Baixo Tocantins - PA, no período de 2007-2015.

\begin{tabular}{|c|c|c|}
\hline Características sócio-demográficas & $\mathbf{N}$ & $\%$ \\
\hline \multicolumn{3}{|l|}{ Gênero } \\
\hline Feminino & 59 & 20.77 \\
\hline Masculino & 225 & 79.23 \\
\hline \multicolumn{3}{|l|}{ Idade } \\
\hline 18 a 27 anos & 100 & 35.21 \\
\hline 28 a 37 anos & 71 & 25.00 \\
\hline 38 a 47 anos & 60 & 21.13 \\
\hline 48 a 57 anos & 29 & 10.21 \\
\hline 58 a 67 anos & 15 & 05.28 \\
\hline$\geq 68$ anos & 9 & 03.17 \\
\hline \multicolumn{3}{|l|}{ Etnia } \\
\hline Amarela & 2 & 00.70 \\
\hline Branca & 21 & 07.39 \\
\hline Parda & 204 & 71.83 \\
\hline Negra & 28 & 09.86 \\
\hline Ignorado & 29 & 10.21 \\
\hline \multicolumn{3}{|l|}{ Escolaridade } \\
\hline Analfabeto & 12 & 04.23 \\
\hline Ensino Fund. Completo & 10 & 03.52 \\
\hline Ensino Fund. Incompleto & 132 & 46.48 \\
\hline Ensino Méd. Completo & 15 & 05.28 \\
\hline Ensino Méd. Incompleto & 10 & 03.52 \\
\hline Ignorado & 103 & 36.27 \\
\hline Não Se Aplica & 2 & 00.70 \\
\hline Total Geral & 284 & 100 \\
\hline
\end{tabular}

Houve maior registro de casos de LV nos municípios de Cametá ( $n=53 ; 18,66 \%)$, Mojú ( $n=42 ; 14,79 \%)$ e Abaetetuba ( $n=41 ; 14,44 \%)$. Quanto à zona de procedência há prevalência da zona rural ( $n=169 ; 59,51 \%)$, em relação a urbana $(n=94 ; 33,10 \%)$ (Tabela 3$)$. 
Tabela 2:Distribuição de casos de Leishmaniose Visceral em adultos na região do Baixo Tocantins- PA, de acordo com o município e zona de procedência, no período de 2007-2015.

\begin{tabular}{|c|c|c|}
\hline Características de moradia & $\mathbf{n}$ & $\%$ \\
\hline \multicolumn{3}{|l|}{ Município de residência } \\
\hline Abaetetuba & 41 & 14.44 \\
\hline Acará & 26 & 09.15 \\
\hline Baião & 15 & 05.28 \\
\hline Barcarena & 35 & 12.32 \\
\hline Cametá & 53 & 18.66 \\
\hline Igarapé-Miri & 32 & 11.27 \\
\hline Limoeiro do Ajurú & 2 & 00.70 \\
\hline Mocajuba & 16 & 05.63 \\
\hline Moju & 42 & 14.79 \\
\hline Oeiras do Pará & 14 & 04.93 \\
\hline Tailândia & 8 & 02.82 \\
\hline \multicolumn{3}{|l|}{ Zona de procedência } \\
\hline Periurbana & 5 & 01.76 \\
\hline Rural & 169 & 59.51 \\
\hline Urbana & 94 & 33.10 \\
\hline Ignorado & 16 & 05.63 \\
\hline Total Geral & 284 & 100 \\
\hline
\end{tabular}

Os principais sinais e sintomas dos pacientes acometidos pela LV foram: fraqueza ( $n=265 ; 93,31 \%)$, febre ( $n=262 ; 92,25 \%)$, emagrecimento ( $n=248 ; 87,32 \%)$, palidez ( $n=211 ; 74,30 \%)$, aumento do baço ( $n=202$; 71,13\%) e aumento do fígado ( $n=182 ; 64,08 \%)$ (Tabela 4).

Tabela 3: Distribuição dos casos de Leishmaniose Visceral em adultos no período de 2007-2015 de acordo com os sinais e sintomas, na região do Baixo Tocantins - PA.

\begin{tabular}{|c|c|c|c|c|c|c|}
\hline \multicolumn{7}{|l|}{ Sinais e Sintomas } \\
\hline & Sim & $\%$ & Não & \% & Ignorado & \% \\
\hline Aumento do Baço & 202 & 71.13 & 66 & 23.24 & 16 & 05.63 \\
\hline Aumento do Fígado & 182 & 64.08 & 80 & 28.17 & 22 & 07.75 \\
\hline Edema & 73 & 25,70 & 197 & 69.37 & 14 & 04.93 \\
\hline Emagrecimento & 248 & 87.32 & 35 & 12.32 & 1 & 00.36 \\
\hline Febre & 262 & 92.25 & 22 & 07.75 & 0 & 00.00 \\
\hline Fraqueza & 265 & 93.31 & 16 & 05.63 & 3 & 01.06 \\
\hline Hemorragia & 26 & 09.15 & 237 & 83.45 & 21 & 07.40 \\
\hline Icterícia & 70 & 24.65 & 189 & 66.55 & 25 & 08.80 \\
\hline Outros & 118 & 41.55 & 363 & 65.49 & 39 & 13.74 \\
\hline Palidez & 211 & 74.30 & 61 & 21.48 & 12 & 04.22 \\
\hline Tosse & 145 & 51.06 & 132 & 46.48 & 7 & 02.46 \\
\hline Total Geral & 284 & 100 & 284 & 100 & 284 & 100 \\
\hline
\end{tabular}

Em relação ao tempo para o diagnóstico, a maioria dos casos teve um intervalo de 0 a 90 dias $(n=220$; $77,46 \%)$ entre os primeiros sintomas e a notificação aos órgãos responsáveis de acordo com o que é visto na tabela 5.

Tabela 4: Distribuição dos casos de Leishmaniose Visceral em adultos no período de 2007-2015, de acordo com tempo para o diagnóstico, na região do Baixo Tocantins - PA.

\begin{tabular}{|c|c|c|}
\hline \multicolumn{3}{|l|}{ Tempo para o diagnóstico } \\
\hline & $\mathbf{N}$ & $\%$ \\
\hline De 0 a 90 dias & 220 & 77.46 \\
\hline De 90 a 180 dias & 43 & 15.14 \\
\hline De 181 a 270 dias & 13 & 04.58 \\
\hline De 271 a 360 dias & 4 & 01.41 \\
\hline$>360$ dias & 4 & 01.41 \\
\hline Total Geral & 284 & 100 \\
\hline
\end{tabular}

Quanto ao tratamento realizado e os desfechos possíveis, o esquema terapêutico utilizado na maioria dos casos, foi o Antimonial Pentavalente $(n=184 ; 64,79 \%)$, mas em um terço $(30,28 \%)$ essa informação foi ignorada, assim como o registro da dose da medicação ( $n=181 ; 63,73 \%$ ) (Tabela 6). 
Tabela 5: Descrição do tratamento utilizado para Leishmaniose Visceral na região do Baixo Tocantins- PA, no período de 2007-2015.

\begin{tabular}{|c|c|c|}
\hline Descrição do tratamento & $n$ & $\%$ \\
\hline \multicolumn{3}{|c|}{ Tempo para o início do tratamento } \\
\hline De 0 a 30 dias & 73 & 25.70 \\
\hline De 31 a 60 dias & 68 & 23.94 \\
\hline De 61 a 90 dias & 41 & 14.44 \\
\hline De 90 a 120 dias & 10 & 03.52 \\
\hline$>120$ dias & 6 & 02.11 \\
\hline Sem Informação & 86 & 30.28 \\
\hline \multicolumn{3}{|l|}{ Tipo de Droga } \\
\hline Anfotericina B & 7 & 02.46 \\
\hline Antimonial Pentavalente & 184 & 64.79 \\
\hline Outras & 6 & 02.11 \\
\hline Não Utilizada & 10 & 03.52 \\
\hline Ignorado & 77 & 27.11 \\
\hline \multicolumn{3}{|l|}{ Dose de medicação } \\
\hline$\geq 20 \mathrm{mg} / \mathrm{kg} / \mathrm{dia} \mathrm{Sb}+5$ & 60 & 21.13 \\
\hline$\geq 10 \mathrm{E} \leq 15 \mathrm{mg} / \mathrm{kg} / \mathrm{dia} \mathrm{Sb}+5$ & 26 & 09.15 \\
\hline$\geq 15 \mathrm{E} \leq 20 \mathrm{mg} / \mathrm{kg} / \mathrm{dia} \mathrm{Sb}+5$ & 17 & 05.99 \\
\hline Ignorado & 181 & 63.73 \\
\hline Total Geral & 1267 & 100 \\
\hline
\end{tabular}

Quanto ao município onde realizaram o tratamento da LV, Cametá foi a cidade com maior número de casos ( $n=51 ; 17,96 \%)$, seguido de Mojú ( $n=42 ; 14,79 \%)$ e Barcarena ( $n=33 ; 11,62)$. (Tabela 7).

Tabela 6: Municípios onde os pacientes adultos realizaram o tratamento da Leishmaniose Visceral na Região do Baixo Tocantins- PA, no período de 2007-2015.

\begin{tabular}{lll}
\hline \hline Municípios do tratamento & $\mathbf{N}$ & \% \\
\hline \hline Abaetetuba & 26 & 09.15 \\
Acará & 21 & 07.39 \\
Baião & 14 & 04.93 \\
Barcarena & 33 & 11.62 \\
Cametá & 51 & 17.96 \\
Igarapé-Miri & 30 & 10.56 \\
Juruti & 1 & 00.35 \\
Limoeiro do Ajurú & 1 & 00.35 \\
Melgaço & 1 & 00.35 \\
Mocajuba & 14 & 04.93 \\
Mojú & 42 & 14.79 \\
Oeiras do Pará & 14 & 04.93 \\
Tailândia & 8 & 02.82 \\
Sem Informação & 28 \\
\hline Total Geral & $\mathbf{2 8 4}$ \\
\hline \hline
\end{tabular}

Em relação ao desfecho do tratamento utilizado pelos pacientes, obteve-se cura em 42,25\% ( $n=120)$, abandono em $02,46 \%(n=7)$ e óbito por LV em $02,82 \%(n=8)$. Enquanto que óbito por outras causas representou $01,76 \%(n=5)$, transferência $15,85 \%(n=45)$ e em 34,86\% (n=99) essa informação foi ignorada (Tabela 8).

Tabela 7: Desfecho do tratamento nos casos de Leishmaniose Visceral em adultos na região do Baixo Tocantins-PA, no período de 2007-2015.

\begin{tabular}{llll}
\hline \hline Desfecho do tratamento & N & \% \\
\hline \hline Abandono & 7 & 02.46 \\
Cura & 120 & 42.25 \\
Óbito por LV & 8 & 02.82 \\
Óbito por outras causas & 5 & 01.76 \\
Transferência & 45 & 15.85 \\
Ignorado & 99 & 34.86 \\
\hline \hline Total Geral & $\mathbf{1 2 6 7}$ \\
\hline \hline
\end{tabular}




\section{DISCUSSÃO}

A mobilização de pessoas e as importantes modificações ambientais, entre outros fatores, estão interferindo na dinâmica do processo de adoecimento, sobretudo nas doenças cujo ciclo de transmissão envolve vetores.

A pesquisa de Silva et al. (2013) realizada em todo o Estado do Pará demonstrou que os maiores números de casos de LV estavam localizados nos municípios de Abaetetuba, Barcarena, Igarapé-Miri, Mojú e Tailândia, todos pertencentes ao Baixo Tocantins. A atual pesquisa identificou maior número de casos nos municípios de Cametá (18,66\%), Mojú (14,79\%), Abaetetuba (14,44\%), Barcarena (12,32\%) e Igarapé-Miri $(11,27 \%)$, respectivamente.

Para alguns estudiosos são diversos os fatores que propiciam a urbanização da LV, tais como, o desmatamento, urbanização progressiva, modificações ambientais causadas pela ação antrópica e por movimentos migratórios, ocupação urbana não planejada, junto com o saneamento deficiente, fazendo com que está endemia apresente, hoje, um novo modelo de distribuição geográfica (FURTADO et al., 2015; REIS et al., 2017).

Tais considerações podem ser reflexos da instalação de medidas progressistas para o desenvolvimento regional, como abertura de estradas, instalação de indústrias e pecuária, com destaque ao Projeto Alça Viária que integrou a área de Baixo Tocantins a Região Metropolitana de Belém e ao Sudeste do Pará, aumentando o fluxo de pessoas, de carros, de cargas, e deslocando parte da população para junto das rodovias onde crescia a atividade agropecuária e madeireira (BASTOS et al., 2010).

Um exemplo de tal fato pode ser observado na pesquisa de Teles et al. (2014), o qual demonstrou no município de Barcarena, entre 2004 a 2008, que a relação de casos de LV urbana/rural foi crescente, saindo de 58,82\% no ano de 2004 para quase $70 \%$ em 2008, e agora nesta pesquisa, a relação urbana/rural dos casos notificados foi de $82,35 \%$, demostrando que a LV na região do Baixo Tocantins continua em processo de urbanização em alguns municípios de uma forma mais acelerada que em outros.

Em outras áreas endêmicas do país o processo de urbanização da LV já é observado há mais tempo. Os estudos de Reis et al. (2017) e Coutinho et al. (2017) também respaldam a atual pesquisa, pois mostraram que a maioria dos casos ocorreram na região urbana, $72 \%$ e $88 \%$, respectivamente. Segundo Werneck et al. (2008) não foi possível afirmar que a transmissão urbana seja diferente daquela em áreas rurais.

Em relação aos pacientes, sabe-se que as crianças e adolescentes são os mais acometidos clinicamente, seguidos pelos adultos jovens (COUTINHO et al., 2017). Analisando está casuística de maiores de 18 anos observou-se que predominaram os casos entre 18-47 anos, do mesmo modo que encontraram Silva et al. (2017) em Pernambuco, onde 68\% dos seus pacientes estavam na faixa etária entre 20-59 anos. A maior incidência, entre adultos, pode ser explicada por representar a população mais ativa realizando atividades na agropecuária e extrativista, portanto, mais exposta ao vetor. De um modo geral, o maior número de pacientes com LV se concentra no gênero masculino em até $60 \%$ dos casos (BRASIL, 2014c), fato observado neste estudo, assim como por Coutinho et al. (2017) que também teve mais de 60\% dos casos no 
gênero masculino.

Para Silva et al. (2013) tal situação deve-se a questão da inserção do homem na zona rural, sendo o que mais sai de casa em busca do alimento e do sustento da família. Esta prática cria então um elemento de risco para este grupo, com maior exposição ao ambiente silvestre, aumentando certamente a probabilidade do contato com o vetor da doença.

A incidência da LV em relação a raça está na dependência da característica populacional onde se apresenta. Na avaliação de casos por Ortiz et al. (2015) em São Paulo, predominaram os casos na raça branca, enquanto neste estudo houve predomínio entre os pardos (71,83\%), seguido da raça negra $(09,86 \%)$. A raça parda envolve a maioria da população no Estado do Pará (IBGE, 2010).

Sendo a LV uma endemia que predomina em áreas de miséria e pobreza, acomete uma parcela da população que apresenta um baixo nível de escolaridade, o que é reforçado praticamente em todos os estudos epidemiológicos realizados, incluindo este, onde aqueles com ensino fundamental incompleto alcançaram taxa de 46,48\%. O mesmo foi visto no estado do Ceará por Cavalcante et al. (2014) (57,7\%), e a situação se agrava nas casuísticas de Coutinho et al. (2017) e de Ortiz et al. (2015) com maioria dos pacientes apresentando escolaridade até o pré-escolar em $55,60 \%$, e $24,60 \%$, respectivamente.

A evolução clínica da LV comporta-se com as clássicas manifestações da doença: febre, fraqueza, emagrecimento, palidez, hepatoesplenomegalia, de evolução insidiosa (SHAFIRI et al., 2017). Do mesmo modo, na área do Baixo Tocantins, observou-se que as manifestações clínicas seguiram as estas perspectivas clínicas, onde os principais sintomas foram a febre, a fraqueza e o emagrecimento, nesta ordem de ocorrência, compatível com as observações de Ortiz et al. (2015). Rigo et al. (2009) relatam que a hepatoesplenomegalia e febre foram os sintomas mais incidentes em seus pacientes. Além disso, a estruturação dos serviços de saúde é necessária para otimizar a vigilância epidemiológica, com medidas de controle vetorial e do adoecimento de cães (REIS et al., 2017).

A medicação mais utilizada nos casos do Baixo Tocantins foi o Antimonial Pentavalente, em 64,79\% dos pacientes, a qual é considerada medicação de escolha para o tratamento (BRASIL, 2014c). Assim também foi usado por Coutinho et al. (2017) em $62,40 \%$ de seus pacientes, obtendo taxa de cura de $59 \%$, superior ao estudo ora realizado, com taxa de cura de $42,25 \%$, do mesmo modo que o observado nos estudos de Cavalcante et al. (2014) com mais de 70\%, e Ortiz et al. (2015) de 90,30\%.

Entretanto, uma parcela considerável dos pacientes $(15,85 \%)$ foi transferida para outros municípios, perdendo-se a informação do desfecho. Além disso, em 34,86\% dos casos, neste tópico na ficha de notificação foi preenchido como ignorado, o que compromete o conhecimento da real situação do desfecho dessa doença na região, assim como, o planejamento de medidas de atuação direta e mais específica para os pacientes acometidos.

\section{CONCLUSÕES}

A pesquisa realizada sobre LV por longo período na área de maior prevalência do Estado do Pará tem compatibilidade clínica e epidemiólogica com os estudos nacionais. Do mesmo modo, mostra um 
comportamento ecoepidemiológico da perspectiva que está se consolidando com a tendência da urbanização dos agravos. Embora esta tendência tenha se consolidado em alguns municípios, a de outros ainda estão aquém desta perspectiva, como o que foi visto em Moju, que apresentou uma relação urbano/rural de 16,67\%. Assim, é de suma importância melhor avaliar os municípios que não seguem a atual tendência para conhecer os aspectos que justificam a manutenção dos casos na zona rural.

Foi possível delinear os aspectos do perfil clínico, sócio-demográfico, e os relacionados ao tratamento da LV na Região do Baixo Tocantins-Pará, considerada área endêmica do agravo, onde nos 9 anos avaliados, foram notificados 284 casos em maiores de 18 anos correspondendo $22,42 \%$ do total, com média de 31 casos ao ano; sendo maior em 2008 (47 casos) e 2011(41 casos), observando-se a partir daí uma tendência a redução das notificações; Prevaleceram os casos entre 18-27 anos (35.21\%), masculinos (79,23\%), pardos (71,23\%), com ensino fundamental incompleto (60.15\%) e moradores de área rural (59,51\%); A distribuição de casos nos municípios foi: Cametá (18,66\%), Moju (14,79\%), Abaetetuba $(14,44 \%)$, Barcarena $(12,32 \%)$, Igarapé-miri (11,27\%), Acará (09,15\%), Mocajuba (05,63\%), Baião $(05,28 \%)$, Oeiras do Pará $(04,93 \%)$, Tailândia (02,82\%) e Limoeiro do Ajuru (00,70\%). Destaca-se Cametá que apresentou o maior registro de casos $(18,66 \%)$, e de tratamento de casos $(17,96 \%)$; O quadro clínico foi composto pelos sinais e sintomas descritos: fraqueza $(93,31 \%)$, febre $(92,25 \%)$, emagrecimento $(87,32 \%)$, palidez $(74,30 \%)$, aumento do baço $(71,13 \%)$, aumento do fígado $(64,08 \%)$, tosse $(51,06 \%)$, edema $(25,70 \%)$, icterícia $(24,65 \%)$, hemorragia (09,15\%); O tempo para o diagnóstico foi de 0-90 dias (64,79\%) e para o início do tratamento foi de 0 a 30 dias $(25,70 \%)$, e a droga mais utilizada foi o Antimonial Pentavalente $(64,79 \%)$; Quanto ao desfecho, observou-se que $42,25 \%$ dos casos evoluíram para cura, $15,85 \%$ foram transferidos para outros municípios, 02,82\% evoluíram a óbito por LV, $02,46 \%$ dos casos abandonaram o tratamento e $01,76 \%$ evoluíram a óbito por outras causa.

Com base nisso, conclui-se que é necessário levar todo este conhecimento obtido no passado e no presente para a comunidade, especialmente para aqueles que atuam na Atenção Básica da Saúde, que estão mais próximas às famílias, e que devem ficar alertas a possibilidade de LV, frente aos casos febris prolongados. Com objetivo de diagnosticar precocemente os casos, realizar tratamento precoce, e consequentemente, diminuir o número de desfechos desfavoráveis da LV no Estado.

\section{REFERÊNCIAS}

ALVAR, J.; VÉLEZ, I. D.; BERN, C.; HERRERO, M.; DESJEUX, P.; CANO, J.. Leishmaniasis worldwide and global estimates of its incidence. PLoS One, São Francisco, v.7, n.5, p.35671, 2012.

BASTOS, A. P. V.; ALMEIDA, O.; CASTRO, E. R.; MARIN, R. A.; PIMENTEL, M. S.; RIVERO, S.. Economia e Sociedade na Região do Tocantins, Pará. Papers do NAEA, n.259, 2010.

BRASIL. Ministério da saúde. Casos confirmados de Leishmaniose Visceral, Brasil, Grandes Regiões e Unidades Federadas. 2000 a 2015. Brasília: Ministério da saúde, 2014a.
BRASIL. Ministério da saúde. Óbitos de Leishmaniose Visceral, Brasil, Grandes Regiões e Unidades Federadas. 2000 a 2015. Brasília: Ministério da saúde, 2014b.

BRASIL. Ministério da Saúde. Manual de vigilância e controle da leishmaniose visceral. Brasília: Ministério da saúde, 2014c.

BRASIL. Ministério do Desenvolvimento Agrário. Plano territorial de desenvolvimento rural sustentável do Baixo Tocantins. Brasília: Ministério da saúde, 2015.

CAVALCANTE, I. J. M.; VALE, M. R.. Aspectos epidemiológicos da Leishmaniose Visceral (Calazar) no Ceará no período de 
2007 a 2011. Rev. Bras. Epidemiol., São Paulo, v.17, n.4, p.911-924, 2014. DOI: https://doi.org/10.1590/1809$\underline{4503201400040010}$

COUTINHO, J. S. V. C.; SANTOS, F. S.; RIBEIRO, R. S. P.; OLIVEIRA, I. B. B.; DANTAS, V. B.; SANTOS, A. B. F. S.. Visceral leishmaniasis and Leishmaniasis- HIV coinfection: comparative study. Rev. Soc. Bras. Med. Trop., Uberaba, v.50, n.5, p.670-674, 2017. DOI: http://dx.doi.org/10.1590/0037-8682-0193-2017

FURTADO, A. L.; NUNES, F. B. B. F.; SANTOS, A. M.; CALDAS, A. J. M.. Análise espaço-temporal da leishmaniose visceral no estado do Maranhão, Brasil. Ciência \& Saúde Coletiva, v.20, n.12, p.3935-3942, 2015. DOI: https://doi.org/10.1590/1413-812320152012.01672015

IBGE. Instituto Brasileiro de Geografia e Estatística. Censo demográfico 2010. Rio de Janeiro: IBGE, 2010.

MACHADO, C. J. S.; SILVA, E. G.; VILANI, R. M.. O uso de um instrumento de política de saúde pública controverso: a eutanásia de cães contaminados por leishmaniose no Brasil. Saúde Soc., São Paulo, v.25, n.1, 2016.

OPAS. Organização Pan-Americana da Saúde. Leishmaniose: Informe Epidemiológico das Américas. Washington, 2017.

ORTIZ, R. C.; ANVERSA, L.. Epidemiologia da leishmaniose visceral em Bauru/SP. Epidemiol. Serv. Saúde, Brasília, v.24, n.1, p.97-104, 2015.

REIS, L. L.; BALIEIRO, A. A. S.; FONSECA, F. R.; GONÇALVES, $M$. J. F.. Changes in the epidemiology of visceral leishmaniasis in Brazil from 2001 to 2014. Rev. Soc. Bras. Med. Trop., Uberaba, v.50, n.5, p.638-645, 2017.

RIGO, R. S.; RIGO L.; HORNER, M. R.. Aspectos clínicos e laboratoriais da Leishmaniose Visceral Americana. J. Bras. Nefrol. São Paulo, v.31, n.1, p.48-53, 2009.
SANCHES, L. C.; MARTINI, C. C.; NAKAMURA, A. A.; SANTIAGO, M. E. B.; LIMA, B. D. L.; LIMA, V. M. F.. Natural canine infection by Leishmania infantum and Leishmania amazonensis and their implications for disease control. Revista Brasileira de Parasitologia Veterinária, Jaboticabal, São Paulo, v.25, n.4, p.465-469, 2016. DOI: https://doi.org/10.1590/S1984-29612016071

SHAFIRI, I.; AFLATOONIAN, M. R.; DAEI-PARIZI, M. H.; HOSSEININASAB, A.; MOSTAFAVI, M.; BAMOROVAT, M. Visceral Leishmaniasis in Southeastern Iran: A Narrative Review. Iran J. Parasitol., Teerã, v.12, n.1, p.1-11, 2017.

SILVA, E. S.; GAIOSO, A. C. I.. Leishmaniose Visceral no Estado do Pará. Rev. Para. Med., Belém, v.27, n.2, 2013.

SILVA, J. A. O.; CARVALHO, G. A.; FAUSTINO, M. A. G. Dinâmica da leishmaniose visceral humana no município de Goiana/PE. PUBVET. Maringá, v.11, n.12, p.1293-1297, 2017.

SILVA JUNIOR, J. N.; GUIMARÃES, F. G.. Expansão e urbanização da Leishmaniose Visceral Humana: caracterização dos fatores determinantes no processo de transmissão em área urbana no município de Rondonópolis/MT (2003 - 2012). Dissertação (Mestrado em Ciências Ambientais e Saúde) - Pontifícia Universidade Católica de Goiás, Goiânia, 2014.

TELES, E. J. C.; SOARES, D. C.; GASPARETTO, D.; VEIGA, N.; FONTELLES, M. J. P.; GARCEZ, L. M.. Leishmaniose visceral no Município minerário de Barcarena, Estado do Pará, Brasil: evolução de 2004 a 2008 e bases para a vigilância epidemiológica. Rev. Pan-Amaz. Saúde, Ananindeua, v.5, n.2, p.21-27, 2014.

WERNECK, G.; L.; PEREIRA, T. J. C. F.; FARIAS, G. C.; SILVA, F. O.; CHAVES, F. C.; GOUVÊA, M. V.. Avaliação da efetividade das estratégias de controle da leishmaniose visceral na cidade de Teresina, Estado do Piauí, Brasil: resultados do inquérito inicial - 2004. Epidemiol Serv. Saúde, Brasília, v.17, n.2, p.87-96, 2008.

A CBPC - Companhia Brasileira de Produção Científica (CNPJ: 11.221.422/0001-03) detém os direitos materiais desta publicação. Os direitos referem-se à publicação do trabalho em qualquer parte do mundo, incluindo os direitos às renovações, expansões e disseminações da contribuição, bem como outros direitos subsidiários. Todos os trabalhos publicados eletronicamente poderão posteriormente ser publicados em coletâneas impressas sob coordenação da Sustenere Publishing, da Companhia Brasileira de Produção Científica e seus parceiros autorizados. Os (as) autores (as) preservam os direitos autorais, mas não têm permissão para a publicação da contribuição em outro meio, impresso ou digital, em português ou em tradução. 